\title{
Procedure to optimize the intercarrier spacing in superchannels impaired by the cascading of WSS-based ROADMs
}

\author{
Paulo J. Pereira ${ }^{1, *}$, João L. Rebola ${ }^{1,2}$, and Luís G. Cancela ${ }^{1,2}$ \\ ${ }^{1}$ Department of Information Science and Technology, ISCTE-Instituto Universitário de Lisboa, Lisboa, Portugal \\ ${ }^{2}$ Optical Communications and Photonics Group, Instituto de Telecomunicações, Lisboa, Portugal
}

\begin{abstract}
A new procedure is proposed to optimize the intercarrier spacing and wavelength selective switch (WSS) bandwidth for superchannels, using a Monte Carlo simulation. We perform an exhaustive assessment of the optical signal-to-noise ratio (OSNR) penalties due to the optical filtering and intercarrier crosstalk, and concluded that the optimum intercarrier spacing is at most $1.1 \mathrm{GHz}$ larger than the Nyquist bandwidth and the results show how the number of cascaded WSSs influences the intercarrier spacing.
\end{abstract}

\section{Introduction}

In superchannels, the spectrum of the subchannels is tightly packed and the crosstalk between subchannels can become a performance constraint. Furthermore, along its path in the optical network, a superchannel passes through several reconfigurable optical add-drop multiplexers (ROADMs). The cumulative filtering reduces the available bandwidth and leads to signal distortion. The performance degradation induced by the wavelength selective switch (WSS) filtering and the intercarrier crosstalk have been studied jointly [1] but a separate quantification of their performance degradation is useful [2,3]. In this work, we perform an exhaustive assessment of the optical signal-to-noise ratio (OSNR) penalties due to the optical filtering and intercarrier crosstalk between subchannels using different $M$-ary quadrature amplitude modulation ( $M$-QAM) formats, symbol rates, number of traversed WSSs, WSS bandwidths, number of subchannels, intercarrier spacings and roll-off factors. We also provide a procedure to obtain optimized values of the intercarrier spacings and WSS bandwidths that guarantee an OSNR penalty below $1.5 \mathrm{~dB}$ [1] for all subchannels after traversing $20 \mathrm{WSSs}$.

\section{Superchannels with 2 up to 10 carriers}

We consider the WSS analytical model described in [4] and an optical transfer function with a $-3 \mathrm{~dB}$ bandwidth $\mathrm{BW}_{\text {OTF }}=8.5 \mathrm{GHz}$. For the Monte Carlo (MC) simulation, we use 28 and $32.5 \mathrm{GBd}$ signals considering two modulation formats, 4-QAM and 16-QAM. The target BER is $4 \times 10^{-3}$ for $28 \mathrm{GBd}$ and $10^{-2}$ for $32.5 \mathrm{GBd}$ [5]. The transmitted symbols in each simulation run are 131072 for the two modulation formats. Two root raise cosine filters are considered, one at the transmitter and the other at the receiver, with the same roll-off factor $\rho=0.1$. First, we perform the simulation for an edge subchannel, then for a center subchannel and, finally, we combine the results from the two subchannels and find the optimum intercarrier spacing and WSS bandwidth. Hence, the procedure has 3 steps:

1. The WSS bandwidth is set equal to the superchannel bandwidth. For an edge subchannel, by varying the intercarrier spacing, we examine if the OSNR penalty is below $1.5 \mathrm{~dB}$ after traversing $20 \mathrm{WSSs}$. We can increase the WSS bandwidth by $12.5 \mathrm{GHz}$, until we obtain the lowest WSS bandwidth that ensures less than $1.5 \mathrm{~dB}$ OSNR penalty.

2 . For the WSS bandwidth found in step 1 and for a center subchannel, we change the intercarrier spacing and obtain the interval of acceptable intercarrier spacings that provide OSNR penalties below $1.5 \mathrm{~dB}$ after 20 WSSs.

3 . The intersection of the two intervals obtained in steps 1 and 2 provides a new interval. Then, and only for intercarrier spacings in this new interval, we add the OSNR penalties from the edge and center subchannels to obtain the total OSNR penalty and then find the intercarrier spacing that provides the minimum total OSNR penalty.

With the proposed procedure, we find the intercarrier spacing that provides the lowest OSNR penalty, below $1.5 \mathrm{~dB}$, for all subchannels after 20 traversed WSSs and the superchannel uses the lowest WSS bandwidth and, subsequently, has the highest spectral efficiency. In Fig. 1, the total OSNR penalty is presented as a function of the intercarrier spacing, for a superchannel with 10 subchannels, $28 \mathrm{GBd}, \rho=0.1$, a $-6 \mathrm{~dB}$ WSS bandwidth of $300 \mathrm{GHz}$ and a) 4-QAM and b) 16-QAM. We can see that, for low intercarrier spacings, the OSNR penalty increases due to higher crosstalk and the OSNR degradation is practically independent of the number of WSSs traversed. On the other hand, the OSNR penalty increases for higher intercarrier spacings due to WSS filtering-induced distortion and becomes dependent on the number of WSSs traversed. The OSNR degradation is higher for the 16-QAM modulation format than for the 4-QAM. An optimum intercarrier spacing for the subchannels is found at the lowest total OSNR penalty due to a balance between these two effects, for a specified number of cascaded WSSs. 
Table 1. Intercarrier spacing and the lowest OSNR penalty after 20 cascaded WSSs for 28 GBd with $\rho=0.1$, 4-QAM and 16-QAM modulation formats, for a different number of subchannels in the superchannel.

\begin{tabular}{|c|c|c|c|c|c|c|c|c|}
\hline \multirow{2}{*}{$\begin{array}{c}\text { Number of } \\
\text { subchannels }\end{array}$} & \multicolumn{2}{|c|}{$\begin{array}{c}\text { WSS bandwidth } \\
{[\mathrm{GHz}]}\end{array}$} & \multicolumn{2}{c|}{$\begin{array}{c}\text { Intercarrier spacing } \\
{[\mathrm{GHz}]}\end{array}$} & \multicolumn{2}{c|}{$\begin{array}{c}\text { Center subchannel } \\
\text { OSNR penalty [dB] }\end{array}$} & \multicolumn{2}{c|}{$\begin{array}{c}\text { Edge subchannel } \\
\text { OSNR penalty [dB] }\end{array}$} \\
\cline { 2 - 9 } & 4-QAM & $16-\mathrm{QAM}$ & 4-QAM & 16 -QAM & 4-QAM & 16-QAM & 4-QAM & 16-QAM \\
\hline 2 & 75 & 87.5 & 28.3 & 28.9 & - & - & 0 & 0 \\
\hline 4 & 125 & 137.5 & 28.6 & 29.0 & 0.1 & 0 & 0.1 & 0 \\
\hline 5 & 150 & 162.5 & 28.3 & 29.1 & 0.3 & 0 & 1.2 & 0 \\
\hline 6 & 187.5 & 187.5 & 28.7 & 29.0 & 0 & 0 & 0 & 0 \\
\hline 8 & 237.5 & 250 & 28.6 & 29.0 & 0.1 & 0 & 0.6 & 0 \\
\hline 10 & 300 & 300 & 28.9 & 28.6 & 0 & 0.8 & 0 & 0.8 \\
\hline
\end{tabular}

Table 2. Intercarrier spacing and the lowest OSNR penalty after 20 cascaded WSSs for $32.5 \mathrm{GBd}$ with $\rho=0.1$, 4-QAM and 16-QAM modulation formats, for a different number of subchannels in the superchannel.

\begin{tabular}{|c|c|c|c|c|c|c|c|c|}
\hline \multirow{2}{*}{$\begin{array}{c}\text { Number of } \\
\text { subchannels }\end{array}$} & \multicolumn{2}{|c|}{$\begin{array}{c}\text { WSS bandwidth } \\
\text { [GHz] }\end{array}$} & \multicolumn{2}{c|}{$\begin{array}{c}\text { Intercarrier spacing } \\
\text { [GHz] }\end{array}$} & \multicolumn{2}{c|}{$\begin{array}{c}\text { Center subchannel } \\
\text { OSNR penalty [dB] }\end{array}$} & \multicolumn{2}{c|}{$\begin{array}{c}\text { Edge subchannel } \\
\text { OSNR penalty [dB] }\end{array}$} \\
\cline { 2 - 9 } & 4-QAM & $16-\mathrm{QAM}$ & 4-QAM & 16-QAM & 4-QAM & 16-QAM & 4-QAM & 16-QAM \\
\hline 2 & 75 & 87.5 & 33.1 & 33.2 & - & - & 0.3 & 0 \\
\hline 4 & 137.5 & 150 & 32.6 & 33.5 & 0.6 & 0 & 1.4 & 0 \\
\hline 5 & 175 & 187.5 & 32.8 & 33.5 & 0.1 & 0 & 0.1 & 0 \\
\hline 6 & 212.5 & 212.5 & 33.1 & 33.5 & 0 & 0 & 0 & 0.5 \\
\hline 8 & 275 & 287.5 & 32.9 & 33.4 & 0.1 & 0 & 0 & 0 \\
\hline 10 & 337.5 & 350 & 32.7 & 33.5 & 0.3 & 0 & 0.4 & 0 \\
\hline
\end{tabular}

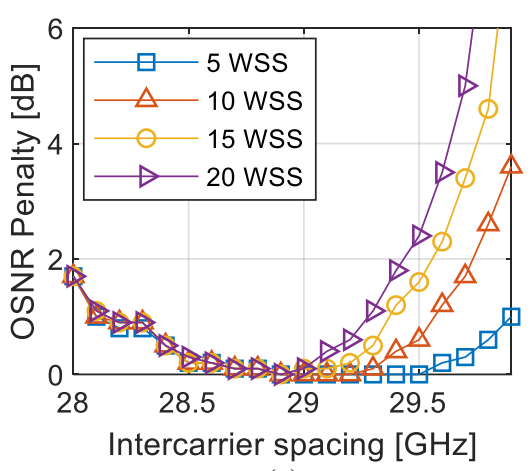

(a)

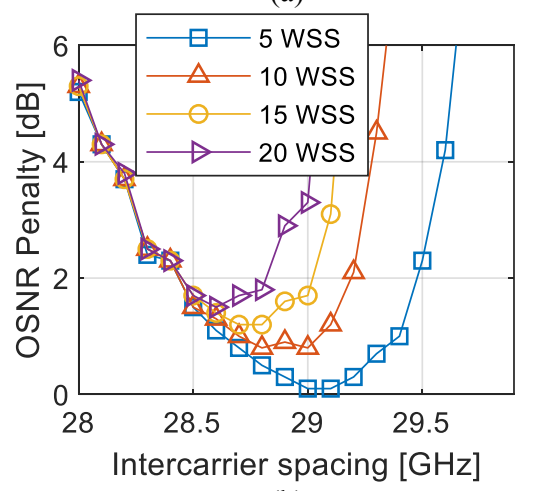

(b)

Fig. 1. Total OSNR penalty dependence on the intercarrier spacing after 5, 10, 15 and 20 WSSs, 10 subchannels, 28 GBd with $\rho=0.1$ and a $-6 \mathrm{~dB}$ WSS bandwidth of $300 \mathrm{GHz}$, for each

WSS in the cascade, for (a) 4-QAM and (b) 16-QAM.

The results obtained from thorough superchannels optimization with a different number of subchannels are presented in Tables 1 and 2, showing the optimum intercarrier spacings and the $-6 \mathrm{~dB}$ WSS bandwidths with the corresponding lowest OSNR penalties for a center and an edge subchannels, for the 4-QAM and 16-QAM modulation formats. For 16-QAM, due to the lower tolerance of higher modulation formats to WSS bandwidth narrowing, we observe almost always a higher intercarrier spacing and almost always a larger WSS bandwidth. We can see that the optimized intercarrier spacing is at most $1.1 \mathrm{GHz}$ larger than the Nyquist bandwidth. If we increase the number of WSSs, it is necessary to reduce the intercarrier spacing and allow a higher OSNR penalty. If the roll-off factor is increased from 0.1 to 0.3 , we have to increase the WSS bandwidth by one or two slots of $12.5 \mathrm{GHz}$ and adjust the intercarrier spacing to ensure OSNR penalty lower than $1.5 \mathrm{~dB}$ in all subchannels after traversing 20 WSSs.

\section{Conclusions}

In this work, a new method to optimize the WSS bandwidth and intercarrier spacing in superchannels has been proposed and implemented using a MC simulation. The optimization has been performed for different symbol rates, modulation formats, number of subchannels, number of cascaded WSSs and roll-off factors. This method guarantees an OSNR penalty below $1.5 \mathrm{~dB}$ and a superchannel with the highest spectral efficiency.

This work was supported in part by Fundação para a Ciência e a Tecnologia (FCT) from Portugal under the project of Instituto de Telecomunicações UIDB/EEA/50008/2020.

\section{References}

1. G. Bosco, et al., JLT, 29, 56-57, (2011)

2. B. Clouet, , et al., JOCN, 8, A122-A124, (2016)

3. J. Pedro, JOCN, 9, C39-C40, (2017)

4. C. Pulikkaseril, et al., Optics Express, 19, 8460 (2011)

5. A. Morea, et al., JOCN, 7, A293-A295, (2015) 